\title{
Lipotoxicity induced pancreatic beta cell damage is associated with GPR119/MST1/FoxO1 Pathway
}

\section{Type}

Research paper

\section{Keywords}

Apoptosis, pancreas, lipotoxicity, FOXO1, GPR119, MST1, Pdx1

\begin{abstract}
Introduction

G-protein-coupled receptor 119 (GPR119) is emerging as a potential therapeutic target against type 2 diabetes with beneficial effects on glucose homeostasis. However, the function of GRP119 in lipotoxicity induced pancreatic beta cell apoptosis and the molecular mechanism remains largely unknown.
\end{abstract}

\section{Material and methods}

Impact of GPR119 on pancreatic islet beta cell apoptosis was evaluated in INS-1 cells treated with palmitate. The subsequent modulation of the MST1-FOXO1-Pdx1 signaling pathway and proapoptotic caspase- 3 system were determined by measuring the target protein and mRNA expression. Dyslipidemia mice with gain and loss of GPR119 function by the application of specific lenti-viral vector was utilized to evaluate the impact of GPR119 on pancreas function in vivo. Lipid metabolism, glucose and insulin response, morphological changes as well as activation/inhibition of MST1-FOXO1-Pdx1 signaling pathway in pancreas were analyzed systematically.

\section{Results}

Palmitate treatment stimulated pro-apoptotic response in INS-1 cells, accompanied by inhibition of GPR119 expression and the subsequent activation of the MST1-FOXO1 combined with inhibition of Pdx1 signaling cascade. Activation of GPR119 by MBX prevents INS-1 cell from lipotoxicity induced apoptosis by targeting the MST1-FOXO1-Pdx1 pathway. Moreover, overexpression of GPR119 significantly attenuates the dyslipidemia and dysfunction of the pancreas. In contrast, inactivation of GPR119 by lentiviral vector in mice results in accelerated pancreas apoptosis and malfunction. The protective effects of GRP119 on lipotoxicity induced pancreas dysfunction are associated with modulating the MST1-FOXO1-Pdx1 signaling cascade.

\section{Conclusions}

GPR119 alleviates lipotoxicity induced pancreatic beta cell apoptosis and malfunction through regulating MST1-FOXO1-Pdx1 signaling pathway. 


\section{Lipotoxicity induced pancreatic beta cell damage is associated with GPR119/MST1/FoxO1 Pathway}

\section{Abstract}

Introduction: G protein-coupled receptor 119 (GPR119) is a potential therapeutic target for type 2 diabetes (T2D) with beneficial effects on glucose homeostasis. However, the function and molecular mechanism of GRP119 in lipotoxicity induced pancreatic beta cell apoptosis cemain undetermined.

Material and methods: Effect of GPR119 on pancreatic islet beta cell apoptosis was evaluated in INS-1 cells treated with palmitate. The subsequent modulation of the MST1-FOXO1-Pdx1 signaling pathway and cell apoptosis were determined. Dyslipidemia mice with overexpression or knockdown GPR119 were utilized to evaluate the impact of GPR119 on pancreas function vivo. Lipid metabolism, glucose and insulin response, morphological changes as well as activation/inhibition of MST1-FOXO1-Pdx1 signaling pathway in pancreas were analyzed systematically.

Results: Palmitate treatment stimulated pro-apoptotic response in INS-1 cells, accompanied by inhibition of GPR119 expression, activation of the MST1-FOXO1 pathway and inhibition of Pdx1 signaling cascade. Activation of GPR119 by MBX treatment prevented INS-1 cells from lipotoxicity induced apoptosis by targeting the MST1-FOXO1-Pdx1 pathway. Moreover, overexpression of GPR119 significantly attenuated dyslipidemia and dysfunction of the pancreas. In contrast, inactivation of GPR119 in mice resulted in accelerated pancreas apoptosis and malfunction. The protective effects of GRP119 on lipotoxicity induced pancreas dysfunction were associated with modulating the MST1-FOXO1-Pdx1 signaling pathway.

\section{Conclusions: GPR119 alleviated lipotoxicity induced pancreatic beta cell apoptosis and} malfunction through regulating MST1-FOXO1-Pdx1 signaling pathway.

Key words: lipotoxicity, apoptosis, GPR119, MST1, FOXO1, Pdx1 pancreas. 


\section{Introduction:}

The dysfunction of pancreatic beta cells is the major cause of type 2 diabetes (T2D) and its complications [1-3]. Lipotoxicity, frequently results from prolonged exposure to the free fatty acids (FFA) such as palmitate, impairs the secretion capacity of insulin and induces apoptosis

in beta cell $[4,5]$. Elevated plasma FFAs levels are observed in obesity and type 2 diabetes, with subsequent lipid accumulation and insulin resistance in target tissues [6]. A series of G proteincoupled receptors (GPCR), including GPR119, GPR40, GPR41, GPR43, GPR84 and GPR120,

have been identified as FFA specific receptors [7]. FFAs exert different effects on insulin secretion from beta cells. Acute exposure to FFAs stimulates insulin secretion, whereas chronic exposure impairs insulin secretion and increase pancreatic beta cell apoptosis. The dual and opposing effects of FFAs on insulin secretion indicate that FFAs might contribute to both hyper- and hypo-insulinemia during the development of T2D. Therefore, GPCRs which recognize fatty acids and activate the intracellular signaling cascades are of particular important in the treatment of T2D [8].

GPR119 is a deorphanized G protein-coupled receptor that is predominantly expressed in pancreatic islets [9] and enteroendocrine cells of the gastrointestinal tract [10]. In pancreatic islets, GPR119 is specifically distributed in beta cells and pancreatic polypeptide cells [11]. GPR119 can be activated by a variety of endogenous ligands, such as oleoylethanolamide, lysophosphatidylcholine and palmitoylethanolamine [12]. The activation of GPR119 stimulates cAMP and $\mathrm{Ca}^{2+}$ release in pancreatic beta cells, enhancing glucose-dependent insulin secretion, thereby decreasing blood glucose in diabetic mice [13,14].

Recently, mammalian STE20 like protein kinase 1 (MST1), also called serine/threonine protein kinase 4, has been identified as an essential regulator for pancreatic beta cell differentiation, proliferation, and apoptosis [15]. MST1 is a core component of the hippo signaling pathway, which can be triggered by a variety of apoptotic stimuli, including oxidative stress, Fas ligand, TNF- $\alpha$, and genotoxic drugs. MST1 is a target and activator of caspases to amplify the apoptotic 
signaling pathway. MST1 promotes cell death through the regulation of multiple downstream

MST1-FOXO1 signaling pathway is associated with cellular oxidative stress and neuronal

apoptosis [17,18]. FOXO1 is mainly expressed in hepatocytes, adipocytes, pancreatic beta

cells and vascular endothelial cells.

FOXO1 directly involves in regulation of physiological activities such as cell oxidative stress, proliferation, and apoptosis. FOXO1 is closely related to and cardiovascular disease [ It has been reported that activated MST1 results in phosphorylation of FOXO1 Ser (212) and promotes apoptosis [20]. Meanwhile, pancreatic beta cell specific FOXO1 transgenic mice were able to maintain beta cell function by increasing the number of beta cells, glucose tolerance and antioxidant capacity.

\section{The expression of FOXO1} mRNA in islet cells of T2D patients was significantly higher than that in non-diabetic controls subjects [2].

The synergistic effect of MST1 and FOXO1 has been proved to play an essential regulatory role in lipid metabolism [21]. Previous study has demonstrated that a high concentration of glucose or fatty acids can cause FOXO1 redistribution in pancreatic beta nuclei [22]. The activation of MST1 can lead to the phosphorylation of FOXO1 and Caspase-3 cleavage [21], indicating that the transcription factor FOXO1 is related to the apoptosis of islet beta cells. However, the specific regulation mechanism still needs further elucidation.

The transcription factor pancreatic duodenal homeobox factor 1 (Pdx1) plays a crucial role in pancreatic development and mature pancreatic beta cell function [23]. Downregulation of Pdx1 expression affects insulin production and secretion, and induce beta cells apoptosis $[24,25]$. Recent studies indicated that MST1 and FOXO1 signaling molecules can regulate pdx1 in pancreatic beta cells $[26,27]$. In this study, we aimed to elucidate the molecular mechanism of GPR119 in lipotoxicity induced beta cell malfunction. A novel signaling cascade of GPR119/FOXO1/Pdx1 in lipotoxicity induced islet cell apoptosis was identified in both in vitro and in vivo. Our findings extended the current understanding of the molecular mechanism of pancreatic beta cell apoptosis, which provided guidance for the development of new diagnostic and therapeutic drugs against T2D and obesity. 
Results

85

86

87

88

89

90

91

92

93

94

\section{FFA activated the phosphorylation of MST1 and FOXO1 by inhibiting GPR119.}

\section{To evaluate the effect of FFA on INS-1 cells, INS-1 cells were stimulated with palmitate. As} shown in Figure $1 \mathrm{~A}$ and $1 \mathrm{~B}$, palmitate treatment induced Caspase-3 cleavage in a timedependent manner and cell apoptosis. Interestingly, GPR119 agonist MBX inhibited MST1 phosphorylation and expression, caspase-3 cleavage level (Figure 1C), and attenuated the palmitate-induced INS-1 cell apoptosis and (Figure 1D). Further gene expression analysis evealed that MBX treatment decreased Bax mRNA levels while stimulated Pdx1 and Insulin mRNA expression (Figure 1E). Lipid analysis showed that cellular content of TG and FFA content was significantly decreased in INS-1 cells treated with MBX (Figure 1F and 1G) $(\mathrm{P}<0.05)$. Insulin levels from INS-1 cells was also significantly increased after MBX intervention (Figure 1H). In addition, the intracellular calcium concentration, was significantly increased in MBX treated INS-1 cells (Figure 1I).

To investigate effect of MST1 on possible downstream signaling cascade FOXO1 and Pdx1, INS-1 cells was overexpressed or knocked down MIST. As shown in Figure 2A and 2B, MST1 overexpression stimulated FOXO1 phosphorylation and activation of caspase-3 in palmitatetreated INS-1 cells. As expected, Pdx1 which is negatively regulated by MST1 was downregulated. Therefore, MST1 overexpression alleviated palmitate induced INS-1 cell injury. In contrast, knock down of MST1 resulted in inactivation of the MST1-FOXO1 signaling pathway while upregulation of Pdx1 expression. These findings indicated that FOXO1 and Pdx1 are downstream molecules of MST1 signaling in islet cells.

\section{Calmodulin (CaM) inhibitor CPZ activated MST1-FOXO1 signaling pathway.}

Previous study demonstrated that treatment of CaM antagonist CPZ in pancreatic cells affects the secretion of insulin by inhibition of $\mathrm{Ca}^{2+}$ influx [28]. In the current study, increased calcium concentration was also observed in palmitate treated INS-1 cells with GPR119 activation 
$111 \mathrm{CaM}$ in palmitate induced signaling alterations was evaluated in CPZ treated INS-1 cells. As

112 shown in Figure $3 \mathrm{~A}$ and $3 \mathrm{~B}$, intervention of CaM in palmitate treated INS-1 cells increased the

113 phosphorylation of MST1 and FOXO1, clMST1, activated the apoptotic protein Caspase-3.

114 These results indicated that palmitate induced MST1-FOXO1 signaling pathway was mediated

115 through the $\mathrm{Ca}^{2+}$ and Calmodulin messenger systems (Figure 3).

Overexpression of GPR119 alleviated pancreatic injury in C57BL/6J mice induced by high-fat diet.

After 18 weeks intervention, mice in high-fat diet (HFD) group showed significantly higher body weight gain compared with mice in the low-fat diet (LFD) group (Figure 4A). In response to a bolus of glucose injection, analysis of area under curve indicated that mice in HFD group demonstrated significant glucose intolerance compared with mice in LFD group (Figure 4B). Similarly, in response to a bolus of insulin administration, a slower decrease in blood glucose was observed in HFD group compared with the LFD group, indicating insulin intolerance in these HFD feeding mice (Figure 4C). The GPR119 overexpression mice showed improved lipid and glucose homeostasis (Table 1). Immunohistochemical staining showed that the number of brown granules in GPR119 overexpression group was higher than that in the control group

GPR119 shRNA mice compared with those in GPR119 overexpression group (Figure 4E).

The pathomorphological changes in pancreatic tissues were examined by electron microscopy. More severe damage was observed in pancreatic islet cells in HFD group compared to the LFD group (Figure 5A). The expression of GPR119 also impacted the ultrastructure of pancreatic beta cells. More severe damage was detected in the HFD + GPR119 shRNA group than that in GPR119 overexpression group. The pancreatic cell damage was characterized by irregular cell morphology, nuclear condensation, nuclear chromatin condensation, mitochondria swollen, 
particles in the islet cells were reduced (Figure 5B). The results of morphological experiments indicated that GPR119 could protect pancreatic beta cells from damage in response to excess lipid (Figure 5C)

The impact of GPR119 on pancreatic signaling cascade was evaluated by

determination of the target protein expression. As indicated in Figure 6, HFD induced down-regulation of GPR119 and activation of MST1-FOXO1 signaling. In consistent with caspase-3.

\section{Discussion}

Previous studies demonstrated that activation of GPR119 by its specific agonists enhances intracellular cAMP and GSIS release, and stimulates the secretion of GLP-1 and GIP, and has a certain inhibitory effect on the apoptosis of pancreatic beta cells $[29,30]$. Our study extended the current understanding of the function of GRP119 in islet cell by identifying the novel downstream MST1-FOXO1-Pdx1 signaling cascade. In palmitate induced lipotoxic INS-1 cell model, MBX mediated GPR119 activation notably reduced islet cell apoptosis rate compared with the control group, accompanied by inhibition of MST1 and FOXO1 activation, decreased pathway plays an important role in the process of neuronal apoptosis. insulin release and enterocytes incretins secretion [31]. In pancreatic beta cells, insulin secretion 
Calmodulin (CaM). In accordance with these studies, inhibition of the binding of $\mathrm{Ca}^{2+}$ to $\mathrm{CaM}$ by CPZ in INS-1 cells activated the apoptosis signaling pathway in response to FFA stimulation, accompanied by activated MST1-FOXO1 signaling cascade and down-regulation of Pdx1 expression. These findings indicated that GRP119 might function through $\mathrm{Ca}^{2+}$ to $\mathrm{CaM}$ pathway in islet cells. However, the direct interaction of GRP119 and the calcium pathway still needs further verification in the future studies.

More recently, MST1-FOXO1 pathway and endoplasmic reticulum (ER) stress have been identified to be involved in the pancreatic beta cells malfunction and apoptosis [33,34]. MST1 in pancreases beta cells can be activated by proinflammatory cytokines as well as glucotoxicity and lipotoxicity [35]. MST1 activation enhanced the impairment of pancreatic beta cells by stimulating phosphorylation of stress kinase JNK, apoptotic mediator caspase-3 and intrinsic cell death mediator BIM, and meanwhile inhibiting the predominant pro-survival AKT signaling cascade [36-38]. In addition, the activation of Pdx1, a vital regulator for insulin secretion and beta cell survival through interaction with GLUT-2 and glucokinase, was also inhibited once MST1 activation.

ipotoxicity activated the MST1 phosphorylation and the downstream signaling cascades such as FOXO1, caspase-3 and Pdx1. MST1 was essential for the downstream signaling pathways associated with beta cell apoptosis and malfunction in response to lipotoxicity. In hyperlipidemia mouse models, the activation of the MST1 signaling in the pancreas was correlated with impaired glucose and insulin metabolism, reflected with glucose intolerance, and decreased insulin levels.

Our study extended the current understanding of FFA-MST1-FOXO1-Pdx1-beta cell malfunction pathway by revealing GRP119 as an essential upstream modulator. In our model, lipid loading in INS-1 cells decreased GPR119 expression, which subsequently activated the MST1 signaling pathway. The cleaved MST1 stimulated the caspase-3 cleavage and FOXO1 phosphorylation as well as inhibited $\mathrm{Pdx} 1$ expression, resulting in the acceleration of beta cell death and impaired insulin secretion. The GRP119 modulation might be mediated through the secondary messenger $\mathrm{Ca} 2+$ and CaM systems. 
The impact of GPR119 in the pancreases function was further confirmed in the hyperlipidemia mouse model. After 18 weeks of high-fat feeding, mice developed obesity, insulin resistance, glucose intolerance, and hyperlipidemia. Interestingly, overexpression of GPR119 in these hyperlipidemia mice resulted in a significant decrease in TG, TC and FFA, and a substantial increase in the content of insulin and $\mathrm{C}$ peptide compared to the control mice. Importantly, GPR119 overexpression also significantly alleviated hyperlipidemia induced islet damage and apoptosis, featured by the chromatin condensation, mitochondria swelling and deformation, and decreased cytoplasmic endocrine granules. The protective effects of GPR119 against pancreas cell apoptosis in mice were associated with modulation of dysfunctional MST1-FOXO1-Pdx1 signaling cascade induced by prolonged fatty acids exposure.

In summary, the current study demonstrated that activation of GPR119 substantially attenuated lipotoxicity induced pancreatic beta cell damage. The effects of GPR119 was associated with $\mathrm{Ca}^{2+}$ - calmodulin pathway and the subsequent modulation of MST1-FoxO1-Pdx1 signaling cascade, which affected pancreatic beta cell function and apoptosis, and finally impacted the insulin secretion and glucose metabolism. This study provided an experimental basis for understanding the mechanism of GPR119 in high-fat induced pancreatic beta cell dysfunction, laying the foundation for the study of the mechanism of obesity and pancreatic beta cell damage and malfunction.

\section{Methods}

\section{Cell culture.}

INS-1 cells (SCC207, EMD Millipore, Burlington, MA, USA) were cultured in RPMI 1640 edium supplemented with $10 \%$ fetal bovine serum (FBS; Gibco), $50 \mathrm{IU} / \mathrm{mL}$ penicillin, 50 mg/L streptomycin, 10 mM HEPES, 2 mM L-glutamine, $1 \mathrm{mM}$ sodium pyruvate and $50 \mu \mathrm{M}$ beta-mercaptoethanol. Cells were cultured at $37{ }^{\circ} \mathrm{C}$ in a humidified atmosphere with $5 \% \mathrm{CO}_{2}$. For palmitate treatment, INS-1 cells were plated into 12 well plates and cultured for 24 hours, $50 \mu \mathrm{M}$ PA diluted in fatty acid-free bovine serum albumin (BSA, Sigma-Aldrich, St. Louis, 
MO, USA) was added to the medium and incubated for indicated time points. BSA was used

220 as vehicle control. In selective studies, following $12 \mathrm{~h}$ of PA treatment, INS-1 cells were treated

221 with GPR119 agonist MBX ( $4 \mu \mathrm{M})$ for 10 minutes or CAM antagonist CPZ $(0.5 \mathrm{mM})$ for 60

222 minutes. For MST1 overexpression and knockdown, INS-1 cells were treated with PA (250.0

$223 \mu \mathrm{M})$ for 12 hours and then transfected with MST1 overexpression plasmid or MST1 silencing

224 shRNA plasmid for 72 hours.

225

226

Plasmid Constructs.

To generate MIST1 overexpression plasmid, full-length rat MST1 was amplified from

228 previously constructed plasmid pMD19-MST1 using the following primers: forward: 5'- GCT

229 GGA TCC GCC ACC ATG GAG ACC GTG CAA CTG AGG AAC-3'; reverse; 5'- ATA GCG

230 GCC GCT CAG AAG TTC TGT TGC CCC TCT TCT TGG C-3'. The amplicon was inserted

231 into the pcDNA3.1 vector with the Not I and BamH I recognition sites. To silence MST1,

232 siRNA against rat MST1 (MST1 siRNA: sense, 5'-GAT CCG CTG GTT CTG TAT CCG ATA

233 TTC TCG AGA ATA TCG GAT ACA GAA CCA GCT TTT G-3', anti-sense, 5'-AAT TCA

234 AAA AGC TGG TTC TGT ATC CGA TAT TCT CGA GAA TAT CGG ATA CAG AAC

235 CAG CG-3') was synthesized (Sangon Biotech, Shanghai, China) and inserted into the

236 pGreenPuro vector. To generate lentiviral transfer plasmids containing GRP119 or shGRP119,

237 mouse GRP119 was amplified from pMD19-GRP119 plasmid using the following primers:

238 forward: 5'-GCT CTA GAG CCG CCA TGG AGT CAT CCT TCT CAT TTG GAG TG-3';

239 reverse; 5'-CGG GAT CCG CCA TCG AGC TCC GGA TGG CT-3'. GRP 119 shRNA was

240 annealed (sense, 5'-GCT AGT TAC CTT CCT GTC AGA GTA ACT AGC CCA GAA ATA

241 GCC TTT TTG-3'; anti-sense, 5'-AAT TCA AAA AGG CTA TTT CTG GGC TAG TTA CTC

242 TGA CAG GAA GGT AAC TAG CCC AGA AAT AGC CG-3'). The amplified GRP119

243 and annealed shGRP119 were inserted into PCDH-CMV-MCS-EF1-GFP and pGreen-CMV-

244 coGFP-T2A-PURO-H1 vectors, respectively. The product was transformed into Stbl3

245 chemically-competent E.coli cells, and the plasmids were extracted by Plasmid Midiprep Kit

246 (Qiagen, Germany). 


\section{Transfection.}

249 To overexpress or silence MST1, INS-1 cells were transfected with overexpression or siRNA

250 plasmids using Lipofectamine 2000 (Invitrogen, Carlsbad, CA, USA) according to the

251 manufacturer's instructions. For lentiviral plasmid transfection, constructed lentiviral transfer

252 plasmid, PLP, PLP2 and PLP/VSVG vectors were transfected into 293T cells using

253 Lipofectamine 2000. At 8 hours post transfection, the 293T cells were switched to complete

254 medium and cultured for $48 \mathrm{hrs}$. The supernatant was then centrifuged at $4000 \mathrm{~g}$ at $4^{\circ} \mathrm{C}$ for 10

255 min to remove cell debris and filtered through a 0.45 um filter membrane.

256

257 Realtime PCR.

258 Total RNA was isolated by Trizol (Invitrogen, USA) according to the manufacturer's

259 instructions. The RNA quality was determined by NanoDrop 2000 (Thermo Fisher, USA). The

260 first-strand cDNA was synthesized using First Strand cDNA Synthesis Kit (Invitrogen, USA).

261 Realtime PCR was performed by SYBR Master Mix on the ABI 7500 Realtime PCR system

262 (Applied Biosystems, USA) as previously described [39]. The relative mRNA expression of

263 target genes to endogenous control glyceraldehyde-3-phosphate dehydrogenase (GAPDH) was

264 calculated using the $2^{-\triangle \triangle C T}$ method. The primer sequences were summarized in Table 2.

265

266 Flow cytometry analysis.

267 Apoptosis analysis was carried out as previously described [40]. The treated cells were

268 dissociated by trypsin and washed with PBS. Subsequently, cells were stained with annexin V

269 FITC (Bio-Rad, USA) and ReadiDrop propidium iodide (Bio-Rad, USA) in binding buffer for

$27020 \mathrm{mins}$ in the dark. Stained cells were subjected to flow cytometry analysis using the

271 FACSCalibur system (BD Biosciences, USA). 


\section{Animal experiments.}

All animal experiments were performed following the Guide for the Care and Use of Laboratory Animals, and all the animal experimental protocols were approved by the Institutional Animal Care and Use Committee (IACUC) at Ningxia Medical University, Yinchuan, China (approval No. 2019-011). Mice were housed in laboratory animal center of Ningxia Medical University with a 12-hour light/dark cycle. The specific-pathogen-free (SPF) male nude C57BL/6 mice (6 week-old; $16-19 \mathrm{~g} ; \mathrm{n}=25$ for each group) were fed a LFD containing $10 \% \mathrm{kcal}$ from fat (MD12031, Mediscience, China) or a HFD containing 60\% kcal from fat (MD12033, Mediscience, China) for 18 weeks. For overexpression of GPR119 in the pancreas, $1.0 \times 10^{8}$ plaque-forming units (PFU) of LV-GFP, LV-GPR119, LV-shctrl and LV-shGPR119 ( $n=5$ for each subgroup) were injected into HFD-fed or LFD-fed mice via the tail vein.

\section{Metabolic measurement.}

For the glucose tolerance test (GTT) and insulin tolerance test (ITT), mice were fasted for 4 hours and then were injected with $2 \mathrm{~g} / \mathrm{kg}$ D-glucose or 1IU/kg insulin intraperitoneally. Blood glucose levels were measured at 15, 30, 60, and 90 mins post-injection using a glucometer. Mice were euthanized after 19 weeks of animal experiments. Blood was collected from heart puncture, and plasma was further isolated. Plasma Insulin and C peptide levels were determined by ELISA kits (Thermofisher, USA). Plasma total cholesterol, triglycerides, free fatty acids levels were determined by commercial kits (Wako, Japan).

\section{Immune-histochemistry.}

The pancreas was isolated from experimental mice and fixed with formalin for 48 hours. Briefly, mice were anesthetized with $4 \%$ chloral hydrate and transcardially perfused with PBS and $4 \%$ paraformaldehyde, subsequently decapitated. Tissues were then embedded with paraffin and the tissue blocks were sliced into $5 \mu \mathrm{m}$ paraffin sections. Immunohistochemical staining of 
tissue section were incubated with $3 \% \mathrm{H}_{2} \mathrm{O}_{2}$ for 10 minutes to inactivate the endogenous peroxidase, followed by incubation with primary antibody GPR119 (1:500, AbCam,

301 Cambridge, UK) for 2 hours and secondary antibody HRP-goat anti-rabbit $\operatorname{IgG}(1: 2,000$,

302 Abcam, Cambridge, UK) for 30 minutes at room temperature. Sections were rinsed in cold PBS (5 times, 3 minutes per time), incubated in avidin-biotin complex (Vecta stain Elite ABC Kit, Vector Laboratories) for 1 hour at $20^{\circ} \mathrm{C}$ and rinsed with PBS (3 times, 10 minutes per time). Finally, sections were developed in diaminobenzidine substrate for 5 minutes at $25^{\circ} \mathrm{C}$ and counterstained with hematoxylin. The sections were further differentiated in $0.1 \% \mathrm{HCl}$, dehydrated in gradient alcohol, cleared by xylene and mounted with resinous media. Images were obtained with microscope under bright field

\section{Western blot.}

extracted from INS-1 cells or pancreas tissues using protein lysis buffer (R0010, Solarbio). After centrifugation at 12,000rpm for $30 \mathrm{mins}$, protein was harvested from supernatant and quantified by bicinchoninic acid (BCA reagents, Thermofisher, USA). $50 \mu \mathrm{g}$ cell lysates were re-suspended in SDS sample loading buffer, subjected to 10\% SDS-PAGE gel and transferred onto a PVDF membrane (Millipore, Bedford, MA, USA). After blocking in Tris-buffered saline with $0.2 \%$ of Tween 20 (TBST) containing 5\% w/v non-fat milk for 2 hours at room temperature, membranes were further incubated with specific primary antibodies overnight at $4{ }^{\circ} \mathrm{C}$. The following primary antibodies were used: Caspase-3, phospho-MST1/ MST1, Pdx1 (Cell Signaling, USA), GRP-119 and $\beta$-actin (ab75312, Abcam, USA), phospho-FOXO1 / FoxO1 (Sata Cruz Biotechnology, Dallas, TX, USA), insulin receptor and BAX (Proteintech, Chicago,IL,USA). Following incubation with the specific HRP-conjugated antibody, chemiluminescence signal was detected using ECL (ECL-808-25, Biomiga, USA). The membranes were developed, and immunoblot bands were subjected to relative densitometric analysis. Protein expression was quantified by determining the relative density of target protein 


\section{Statistical analysis.}

329 All results are expressed as the mean \pm SEM. Data between groups was analyzed by one-way 330 or two-way ANOVA, followed by post hoc Tukey's multiple comparison analysis. Differences 331 between groups were considered statistically significant at $P<0.05$. Analysis was conducted 332 using GraphPad Prism 6 software.

\section{Competing interests:}

335 The authors declare that they have no competing interests.

\section{Funding}

This work was supported by the National Natural Science Foundation of China (No. 81670798), the spring plan of Ministry of Education(Z2016039), West China Top Class Discipline Project in Basic Medical Sciences, Ningxia Medical University.

\section{Acknowledgements}

The authors would like to thank Dr. Jiajun Zhao for critical comments.

\section{Author Contributions}

346 Y.Y. and L.C. designed the study. L.R.M., J.N.L, Y.L.S and Y.H.L conducted the experiments.

347 Y.L., Y.W and H.S. analyzed the data. Y.S.L., H.Q. performed the animal experiments. Y.Y.,

348 L.C. and L.R.M. wrote and edited the manuscript. All authors reviewed and approved the content of the paper. 
Ethics approval and consent to participate

352

353

354

355

356

357

358

359

360

361

362

363

364

365

366

367

368

369

370

371

372

373

374

375

376

377

All animal experiments were performed in accordance with the Guide for the Care and Use of Laboratory Animals, and all the animal experimental protocols were approved by the Institutional Animal Care and Use Committee (IACUC) at Ningxia Medical University (Yinchuan, China).

\section{Abbreviations:}

GPR119: G-protein-coupled receptor 119; MST1: mammalian STE20 like protein kinase 1; T2D: type 2 diabetes; FOXO1 (Forkhead box O1); FFA: free fatty acids; Pdx1: pancreatic duodenal homeobox factor 1;CaM: Calmodulin; CPZ: Chlorpromazine; HFD: high fat diet; LDF: low fat diet; PA: palmitate.

\section{Figure legends:}

Figure 1. Palmitate treatment stimulated the phosphorylation of MST1 by inhibiting GPR119 in INS-1 cells. (A). INS-1 cells were treated with Palmitate (PA) $(250 \mu \mathrm{M})$ at indicated time points. The activated caspase-3 expression (cl-Cas 3) was determined by western blot and quantified by relative density to $\beta$-actin. Data are expressed as mean $\pm \operatorname{SEM}(n=5)$. *, $P<0.05$ vs. control. (B, D) Detection of apoptosis rate by flow cytometry. ${ }^{*} \mathrm{P}<0.05$, PA $v s$ control; PA+MBX vs. control+MBX. (C). INS-1 cells were treated with $250 \mu \mathrm{M}$ palmitate for $12 \mathrm{~h}$, followed by treatment of $4 \mu \mathrm{M}$ MBX for $10 \mathrm{~min}$. The expression of GPR119, MST1, pMST1, clMST1 and caspase-3 cleavage (clC3) expression were determined by western blotting and quantified by relative density to $\beta$-actin. Data are expressed as mean $\pm \mathrm{SEM}(\mathrm{n}=$ 5). *, $P<0.05$ vs. control. (E) Target mRNA expression in INS-1 cells treated with MBX. Data are expressed as mean \pm SEM $(n=5)$. (F, G) Effects of GPR119 agonist MBX on cellular TG and FFA contents in INS-1 cells. Data are expressed as the mean $\pm \operatorname{SEM}(n=5)$. (H) Insulin secretion in PA treated INS-1 cells in the presence/absence of MBX at indicated time points. 
(I) Intracellular calcium concentration in INS-1 cells determined by flow cytometry. Data are expressed as the mean \pm SEM $(\mathrm{n}=5) .{ }^{*} P<0.05,{ }^{\#} P<0.01 v s$. control. Experiments were repeated three times.

Figure 2. Target protein expression in PA treated INS-1 cells with gain and loss of MST1 expression. (A) INS-1 cells were treated with PA $(250 \mu \mathrm{M})$ for $12 \mathrm{hrs}$ and then were incubated MST1 overexpression plasmid MST1 or MST1 shRNA plasmid for $72 \mathrm{hrs.} \mathrm{Protein} \mathrm{expression}$ of MST1, pMST1, FOXO1, pFOXO1, clMST1 and caspase-3 cleavage (clC3) was determined by western blot. (B) Quantification of protein bands by densitometry analyses. Data are expressed as the mean $\pm \operatorname{SEM}(\mathrm{n}=5) .{ }^{*} P<0.05,{ }^{\#} P<0.01 v s$. control. Experiments were repeated three times.

Figure 3. Target protein expression in INS-1 cells treated with Palmitate in the presence/ absence of CPZ. INS-1 cells were treated with palmitate $(250 \mu \mathrm{M})$ for $12 \mathrm{~h}$, followed by treatment of CPZ (0.5 mM) for 60 min.(A) The protein levels of MST1, pMST1, FOXO1, pFOXO1, clMST1 and cleaved caspase-3 (clC3) were determined by western blot using specific antibodies. (B). Quantification of the protein bands by densitometry and normalized to $\beta$-actin. Data are expressed as mean $\pm \operatorname{SEM}(n=5) .{ }^{*}<<0.05, \# P<0.01 ; C P Z$ treated vs. control. All data were derived from three independent experiments.

Figure 4. Overexpression of GPR119 alleviated pancreatic injury in C57BL/6J mice induced by high fat feeding. 6-week-old male C57 mice were fed on HFD or LFD for 18 weeks and then were given either LV-GPR119 or LV-shGPR119 by tail vein injection. (A). Body weight of mice on a LFD or HFD. Body weight of the experimental mice were measured weekly and shown as the mean $\pm \operatorname{SEM}(n=25)$. $(\mathbf{B}, \mathbf{C})$. Glucose tolerance and insulin tolerance test in LFD and HFD mice at 12 weeks feeding. (D). GPR119 protein expression in the pancreas of mice determined by IHC $(400 \mathrm{X})$. (E). Pancreas tissue morphological changes were determined by HE staining. Representative images (400X) are shown. Data are expressed as the mean $\pm \operatorname{SEM}(\mathrm{n}=5) .{ }^{*} P<0.05,{ }^{\#} P<0.01$ vs. control.

Figure 5. Pancreas damage of mice on a high fat diet. Mice were fed on HFD or LFD for 18 weeks and then were given either LV-GPR119 or LV-shGPR119 by tail vein injection for 1 
week $(n=5)(A)$. Electron microscope images of pancreatic tissue section of LFD/HFD mice. (B). Electron microscopy images of pancreas tissue on HFD group with GPR119 overexpressing or GPR119 silencing. (a,d X3000; b,e X5000; c,f X15000). (C). Model of pancreas GRP119 function in pancreas high fat milieu. Lipid loading in INS-1 cells decreased GPR119 expression which subsequently activated the MST1 signaling. Active cleaved MST1 then stimulated the caspase-3 cleavage and FOXO1 phosphorylation as well as inhibited Pdx1 activation. Such alteration in the signaling cascade resulted in acceleration of beta cell death and impaired insulin secretion. In response to lipotoxicity, the function of GPR119 is likely through affecting intracellular $\left[\mathrm{Ca}^{2+}\right]$ release.

Figure 6. HFD induced down-regulation of GPR119 and activation of MST1-FOXO1 signaling. (A) Mice were fed on HFD or LFD for 18 weeks and then were given either LV-GPR119, LVGFP, LV-shGPR119 or LV-sh Control by tail vein injection for 1 week. Pancreas was isolated, and protein was extracted. Protein expression of MST1, pMST1, FOXO1, pFOXO1, clMST1 and caspase-3 cleavage (clC3) was determined by western blot. (B, C) Quantification of protein bands by densitometry analyses. Data are expressed as the mean $\pm \operatorname{SEM}(\mathrm{n}=5) .{ }^{*} P<0.05,{ }^{\#} P$ $<0.01$ vs. control. Experiments were repeated three times.

Table 1. Detection of insulin, $C$ peptide and FFA in serum of mice.

\begin{tabular}{lccc}
\hline \multicolumn{1}{c}{ Group } & Insulin (IU/mL) & C peptide $(\mathrm{ug} / \mathrm{mL})$ & FFA (mmol/L) \\
\hline LFD & $19.95 \pm 0.09$ & $0.36 \pm 0.03$ & $35 \pm 3.68$ \\
LFD-LV+GFP & $19.75 \pm 0.37$ & $0.32 \pm 0.02$ & $16 \pm 0.89$ \\
LFD-LV+GPR119 & $14.00 \pm 0.15^{\mathrm{a}}$ & $0.27 \pm 0.01^{\mathrm{a}}$ & $6 \pm 0.73^{\mathrm{a}}$ \\
LFD-LV+shCtrl & $16.63 \pm 0.39$ & $0.30 \pm 0.007$ & $25 \pm 3.13$ \\
LFD-LV+shGPR119 & $15.63 \pm 0.43$ & $0.35 \pm 0.007$ & $35 \pm 8.02$ \\
HFD & $23.52 \pm 0.20$ & $0.28 \pm 0.007$ & $55 \pm 10.6$ \\
HFD-LV+GFP & $17.70 \pm 0.42$ & $0.20 \pm 0.007$ & $15 \pm 1.03$ \\
HFD-LV+GPR119 & $40.1 \pm 0.33^{\mathrm{c}}$ & $0.70 \pm 0.007^{\mathrm{c}}$ & $5 \pm 0.06^{\mathrm{c}}$
\end{tabular}



HFD-LV+shCtrl
$31.77 \pm 0.46$
$0.58 \pm 0.03$
$13 \pm 1.08$
HFD-LV+shGPR119
$41.28 \pm 0.85$
$0.57 \pm 0.03$
$11 \pm 1.32$

424

425

426

427

428

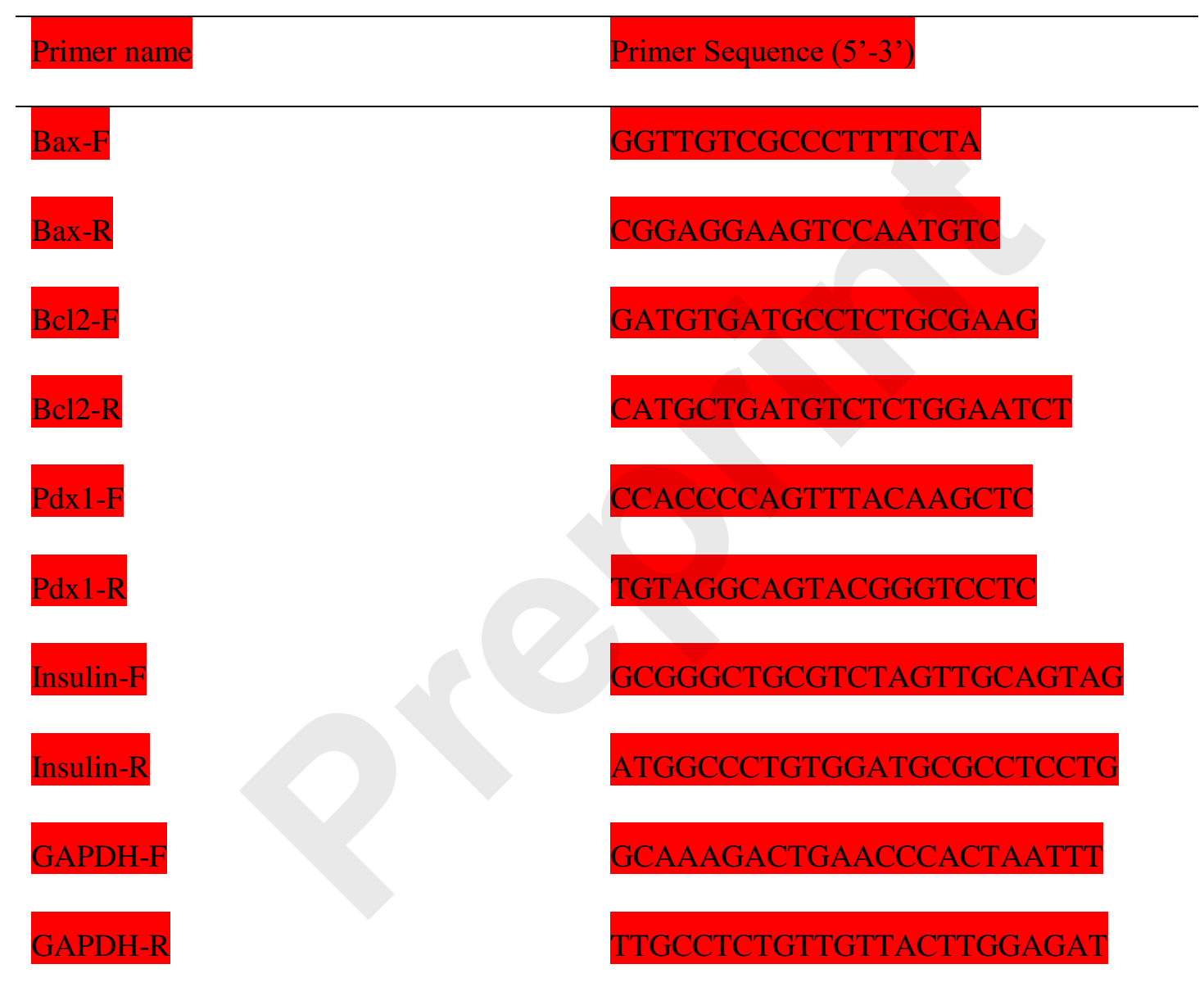

Remarks: a, LFD-LV+GPR119 vs LFD-LV+GFP, P < 0.05; c, HFD-LV+GPR119 vs HFD-

$\mathrm{LV}+\mathrm{GFP}, \mathrm{P}<0.05$.

Table 2. Primers used for Realtime PCR.

Primer name $\quad$ Primer Sequence (5'-3')

Bax-F GGTTGTCGCCCTTTTCTA

Bax-R

CGGAGGAAGTCCAATGTC

Bcl2-F

GATGTGATGCCTCTGCGAAG

ATGCTGATGTCTCTGGAATCT

CACCCCAGTTTACAAGCTC

TGTAGGCAGTACGGGTCCTC

GCGGGCTGCGTCTAGTTGCAGTAG

ATGGCCCTGTGGATGCGCCTCCTG

GCAAAGACTGAACCCACTAATTT

TTGCCTCTGTTGTTACTTGGAGAT
429

430

431

432

433

434

\section{References}

1. Boden G. Role of fatty acids in the pathogenesis of insulin resistance and NIDDM.
Diabetes 1997; 46: 3-10.
2. Del Guerra S, Lupi R, Marselli L, et al. Functional and molecular defects of pancreatic 
3. Ying C, Wang S, Lu Y, et al. Glucose fluctuation increased mesangial cell apoptosis

460

461

462

463

464

465

466

467

468

469

470

related to AKT signal pathway. Arch Med Sci 2019; 15: 730-737.

4. Sharma RB and Alonso LC. Lipotoxicity in the pancreatic beta cell: not just survival and function, but proliferation as well? Curr Diab Rep 2014; 14: 492.

5. Oh YS, Bae GD, Baek DJ, et al. Fatty Acid-Induced Lipotoxicity in Pancreatic BetaCells During Development of Type 2 Diabetes. Front Endocrinol (Lausanne) 2018; 9: 384.

\section{Reimann F and Gribble FM. G protein-coupled receptors as new therapeutic targets for type 2 diabetes. Diabetologia 2016; 59: 229-233.}
8. Piro S, Anello M, Di Pietro C, et al. Chronic exposure to free fatty acids or high glucose induces apoptosis in rat pancreatic islets: possible role of oxidative stress. Metabolism: clinical and experimental 2002; 51: 1340-1347.

9. Soga T, Ohishi T, Matsui T, et al. Lysophosphatidylcholine enhances glucosedependent insulin secretion via an orphan G-protein-coupled receptor. Biochem Biophys Res Commun 2005; 326: 744-751.
10. Chu ZL, Carroll C, Alfonso J, et al. A role for intestinal endocrine cell-expressed g protein-coupled receptor 119 in glycemic control by enhancing glucagon-like Peptide- 1 and glucose-dependent insulinotropic Peptide release. Endocrinology 2008; 149: 2038-2047.
11. Ning Y, O'Neill K, Lan H, et al. Endogenous and synthetic agonists of GPR119 differ in signalling pathways and their effects on insulin secretion in MIN6c4 insulinoma cells. British journal of pharmacology 2008; 155: 1056-1065.

12. Moran BM, Abdel-Wahab YH, Flatt PR, et al. Activation of GPR119 by fatty acid agonists augments insulin release from clonal beta-cells and isolated pancreatic islets and improves glucose tolerance in mice. Biol Chem 2014; 395: 453-464.

13. Dhayal S and Morgan NG. The significance of GPR119 agonists as a future treatment for type 2 diabetes. Drug news \& perspectives 2010; 23: 418-424.

14. Lauffer LM, Iakoubov $\mathrm{R}$ and Brubaker PL. GPR119 is essential for oleoylethanolamide-induced glucagon-like peptide-1 secretion from the intestinal enteroendocrine L-cell. Diabetes 2009; 58: 1058-1066.

15. Ardestani A and Maedler K. MST1: a promising therapeutic target to restore functional beta cell mass in diabetes. Diabetologia 2016; 59: 1843-1849.

16. Bi W, Xiao L, Jia Y, et al. c-Jun N-terminal kinase enhances MST1-mediated pro- 
471

472

473

474

475

476

477

478

479

480

481

482

483

484

485

486

487

488

489

490

491

492

493

494

495

496

497

498

499

500

501

502

503

504

505

506

507

apoptotic signaling through phosphorylation at serine 82 . The Journal of biological

chemistry 2010; 285: 6259-6264.

17. Lehtinen MK, Yuan Z, Boag PR, et al. A conserved MST-FOXO signaling pathway
mediates oxidative-stress responses and extends life span. Cell 2006; 125: 987-1001.

18. Liu P, Kao TP and Huang H. CDK1 promotes cell proliferation and survival via phosphorylation and inhibition of FOXO1 transcription factor. Oncogene 2008; 27 : $4733-4744$.

19. Yuan Z, Lehtinen MK, Merlo P, et al. Regulation of neuronal cell death by MST1 FOXO1 signaling. The Journal of biological chemistry 2009; 284: 11285-11292.

20. Valenti L, Rametta R, Dongiovanni P, et al. Increased expression and activity of the transcription factor FOXO1 in nonalcoholic steatohepatitis. Diabetes 2008; 57: 13551362.

21. Kitamura YI, Kitamura T, Kruse JP, et al. FoxO1 protects against pancreatic beta cell failure through NeuroD and MafA induction. Cell metabolism 2005; 2: 153-163.

22. Wei B, Dui W, Liu D, et al. MST1, a key player, in enhancing fast skeletal muscle atrophy. BMC biology 2013; 11: 12.

23. Jonsson J, Carlsson L, Edlund T, et al. Insulin-promoter-factor 1 is required for pancreas development in mice. Nature 1994; 371: 606-609.

24. Johnson JD, Ahmed NT, Luciani DS, et al. Increased islet apoptosis in Pdx1+/- mice. The Journal of clinical investigation 2003; 111: 1147-1160.

25. Brissova M, Shiota M, Nicholson WE, et al. Reduction in pancreatic transcription factor PDX-1 impairs glucose-stimulated insulin secretion. The Journal of biological chemistry 2002; 277: 11225-11232.

26. Lv L, Chen H, Sun J, et al. PRMT1 promotes glucose toxicity-induced beta cell dysfunction by regulating the nucleo-cytoplasmic trafficking of PDX-1 in a FOXO1dependent manner in INS-1 cells. Endocrine 2015; 49: 669-682.

27. Hennige AM, Ranta F, Heinzelmann I, et al. Overexpression of kinase-negative protein kinase Cdelta in pancreatic beta-cells protects mice from diet-induced glucose intolerance and beta-cell dysfunction. Diabetes 2010; 59: 119-127.

28. Dadi PK, Vierra NC, Ustione A, et al. Inhibition of pancreatic beta-cell Ca2+/calmodulin-dependent protein kinase II reduces glucose-stimulated calcium influx and insulin secretion, impairing glucose tolerance. The Journal of biological chemistry 2014; 289: 12435-12445.

29. Chu ZLL, J. N, Al-Shamma, H.A., Jones, R.M. Combination therapy for the treatment of diabetes and conditions related thereto and for the treatment of conditions ameliorated by increasing a blood glp-1 level International Patent Publication 2009: 762-768. 
508

509

510

511

512

513

514

515

516

517

518

519

520

521

522

523

524

525

526

527

528

529

530

531

532

533

534

535

536

537

538

539
30. Overton HA, Babbs AJ, Doel SM, et al. Deorphanization of a G protein-coupled receptor for oleoylethanolamide and its use in the discovery of small-molecule hypophagic agents. Cell metabolism 2006; 3: 167-175.

31. Flock G, Holland D, Seino Y, et al. GPR119 regulates murine glucose homeostasis hrough incretin receptor-dependent and independent mechanisms. Endocrinology 2011; 152: 374-383.
32. Graves TK and Hinkle PM. $\mathrm{Ca}(2+)$-induced $\mathrm{Ca}(2+)$ release in the pancreatic beta-cell: direct evidence of endoplasmic reticulum $\mathrm{Ca}(2+)$ release. Endocrinology 2003; 144: $3565-3574$.
33. Nakagawa T, Zhu H, Morishima N, et al. Caspase-12 mediates endoplasmic-reticulum- specific apoptosis and cytotoxicity by amyloid-beta. Nature 2000; 403: 98-103.
34. Wellen KE and Hotamisligil GS. Inflammation, stress, and diabetes. The Journal of clinical investigation 2005; 115: 1111-1119.
35. Yuan T, Lupse B, Maedler K, et al. mTORC2 Signaling: A Path for Pancreatic beta Cell's Growth and Function. J Mol Biol 2018; 430: 904-918.
36. Ardestani A, Paroni F, Azizi Z, et al. MST1 is a key regulator of beta cell apoptosis and dysfunction in diabetes. Nat Med 2014; 20: 385-397.
37. Guo H, He Y, Bu C, et al. Antitumor and apoptotic effects of 5-methoxypsoralen in U87MG human glioma cells and its effect on cell cycle, autophagy and PI3K/Akt signaling pathway. Arch Med Sci 2019; 15: 1530-1538.
38. Zhang Y, Zhang R and Ni H. Eriodictyol exerts potent anticancer activity against A549 human lung cancer cell line by inducing mitochondrial-mediated apoptosis, G2/M cell cycle arrest and inhibition of m-TOR/PI3K/Akt signalling pathway. Arch Med Sci 2020; 16: 446-452.
39. Zhao M, Wang K, Shang J, et al. MiR-345-5p inhibits tumorigenesis of papillary thyroid carcinoma by targeting SETD7. Arch Med Sci 2020; 16: 888-897.
40. Zhihong Z, Rubin C, Liping L, et al. MicroRNA-1179 regulates proliferation and chemosensitivity of human ovarian cancer cells by targeting the PTEN-mediated PI3K/AKT signaling pathway. Arch Med Sci 2020; 16: 907-914.

41. Eken MK, Ersoy GS, Kaygusuz EI, et al. Etanercept protects ovarian reserve against ischemia/reperfusion injury in a rat model. Arch Med Sci 2019; 15: 1104-1112. 
A

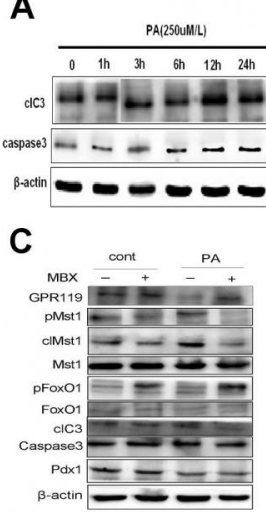

E

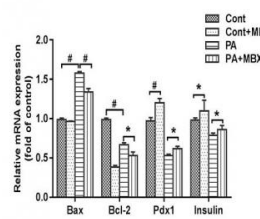

B
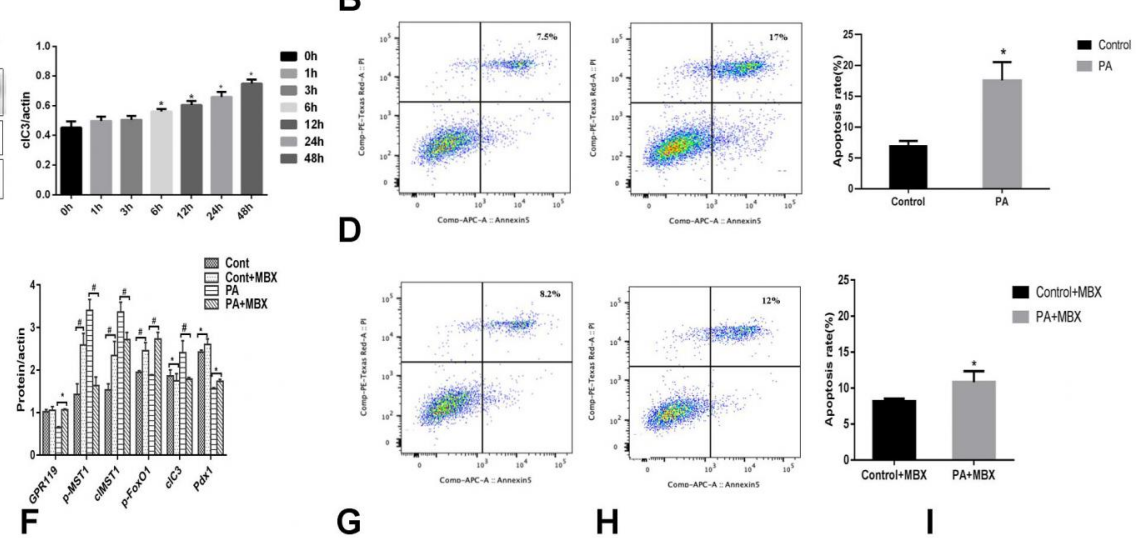

G

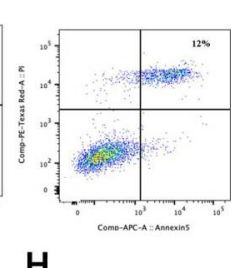

H
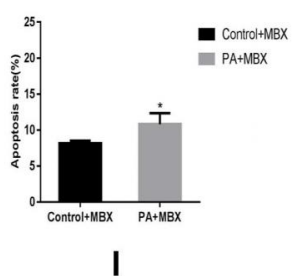

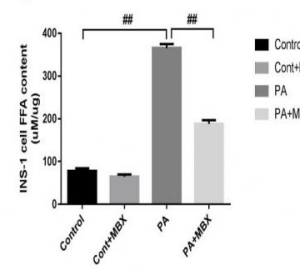

of

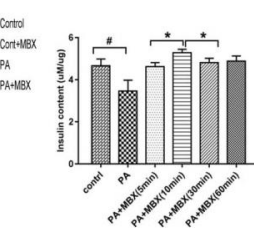

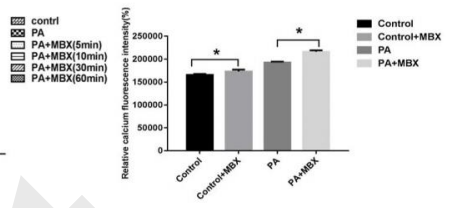


A

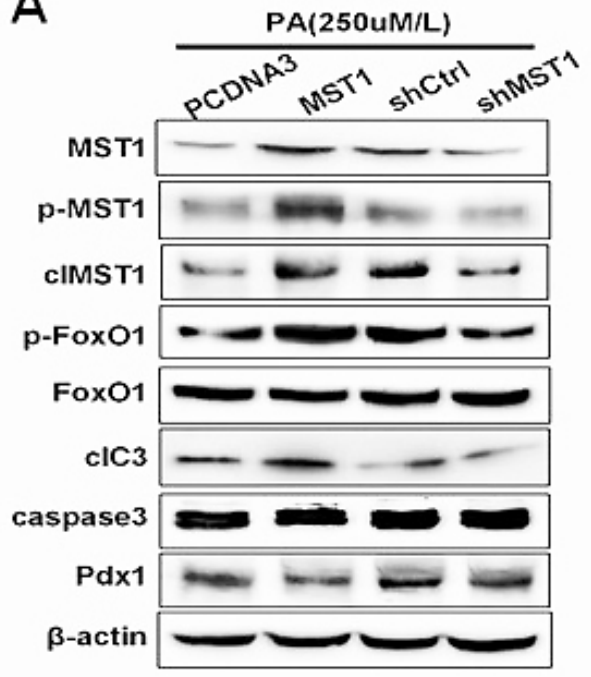

B

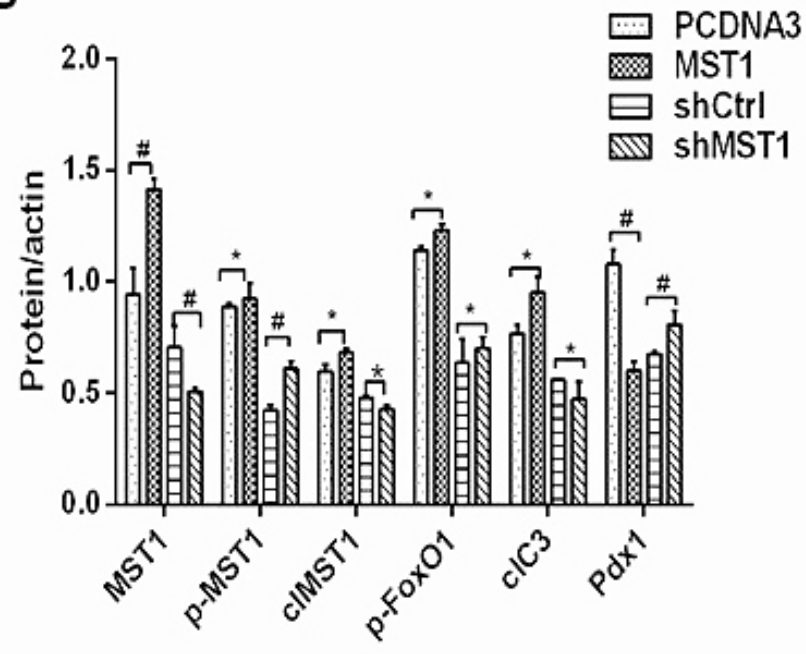


A

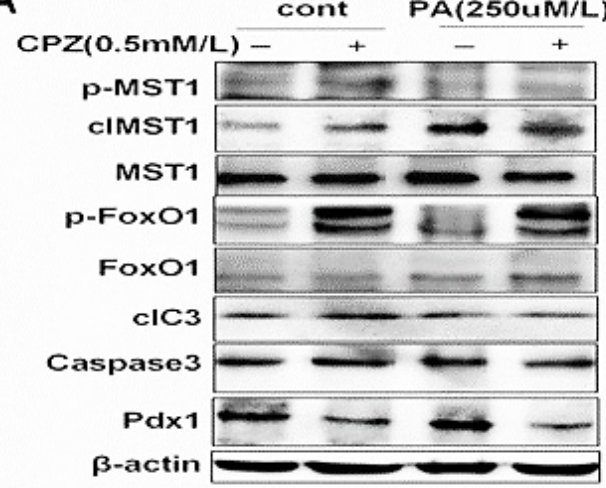

B

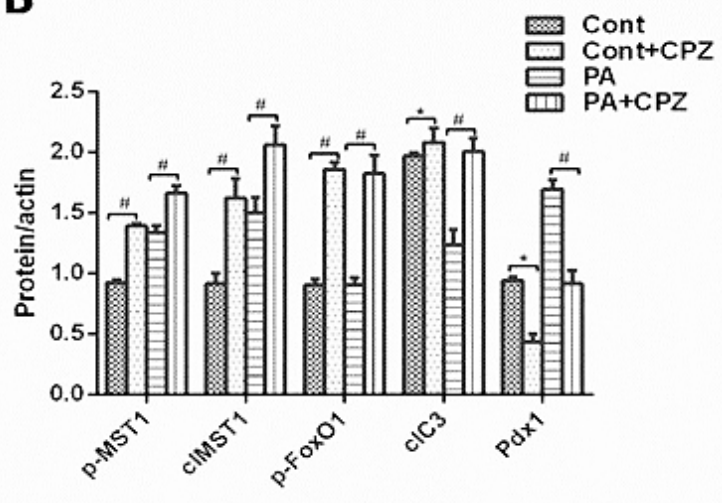



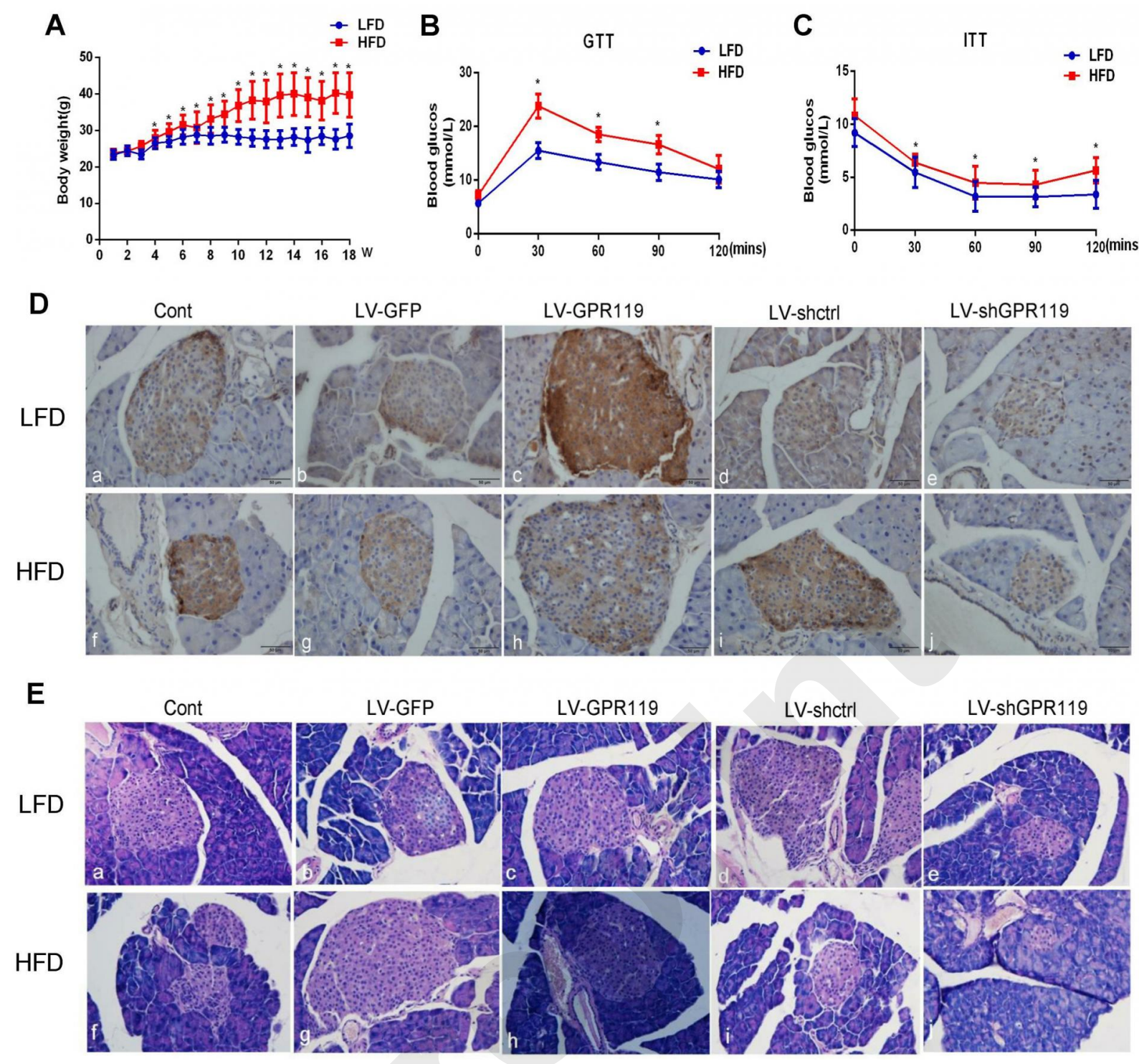


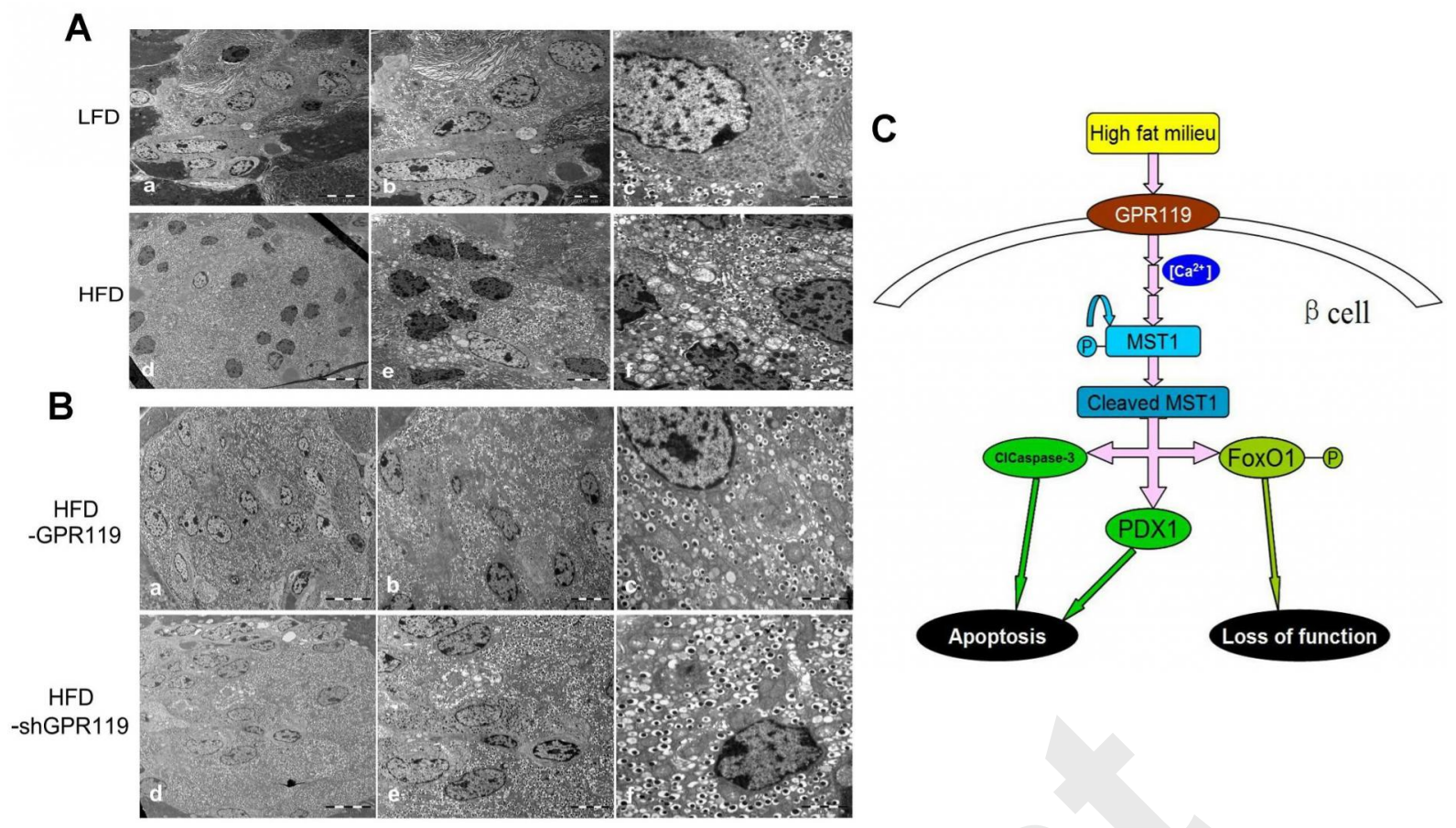



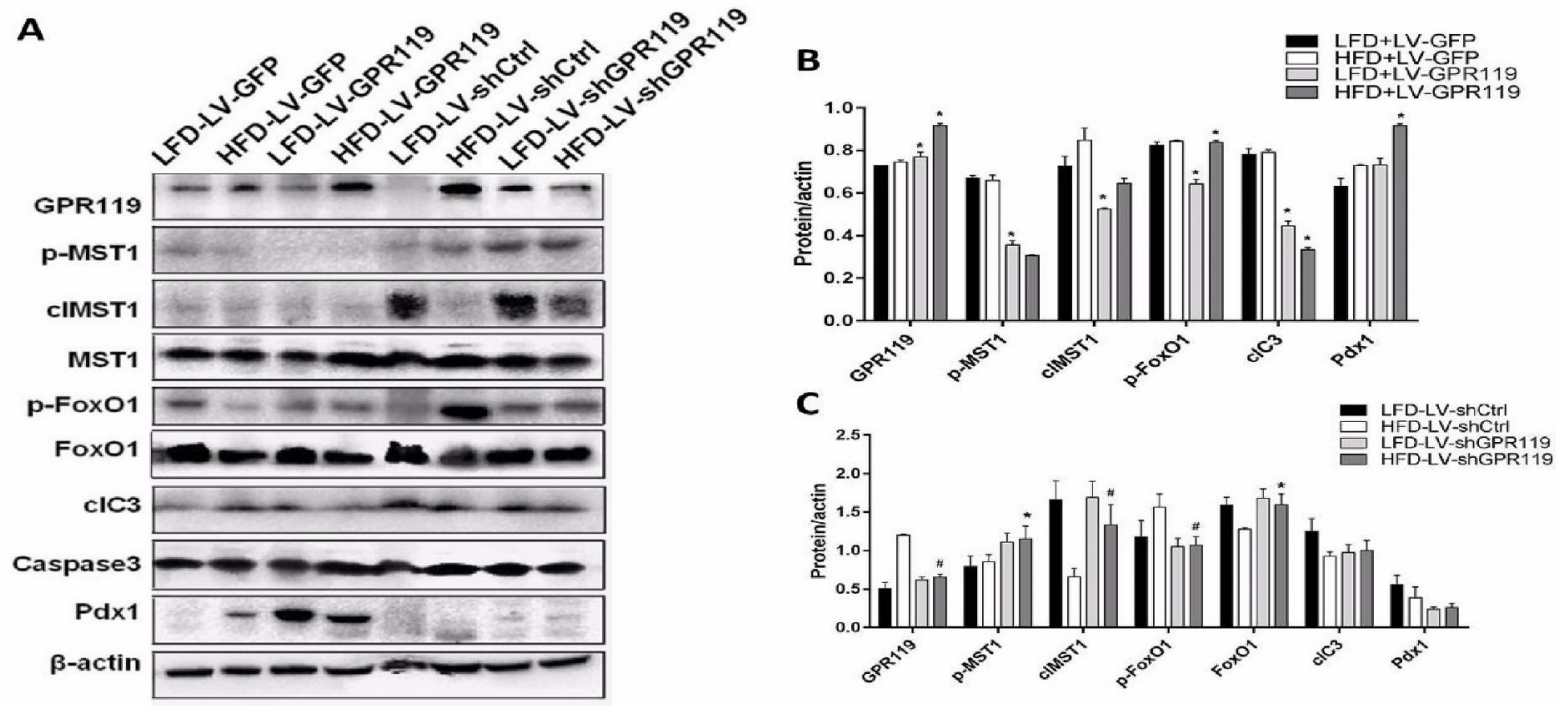